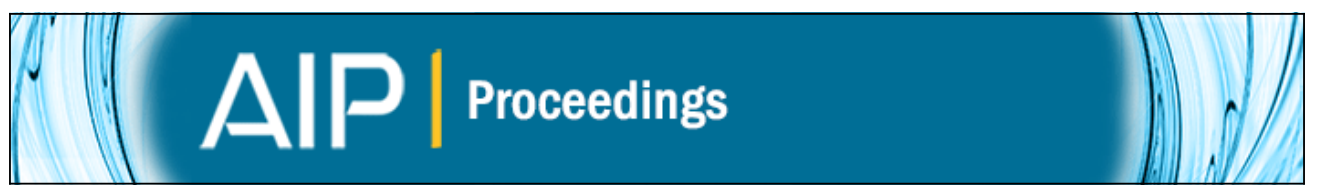

\title{
Fragmentation in the First Galaxies
}

Chalence Safranek-Shrader

Citation: AIP Conference Proceedings 1294, 276 (2010); doi: 10.1063/1.3518875

View online: http://dx.doi.org/10.1063/1.3518875

View Table of Contents:

http://scitation.aip.org/content/aip/proceeding/aipcp/1294?ver=pdfcov

Published by the AIP Publishing

Articles you may be interested in

Supermodel Analysis of Galaxy Clusters

AIP Conf. Proc. 1248, 291 (2010); 10.1063/1.3475237

The Influence of AGN Feedback on Galaxy Cluster Observables

AIP Conf. Proc. 1201, 350 (2009); 10.1063/1.3293074

The Moderate Cooling Flow Model and Feedback in Galaxy Formation

AIP Conf. Proc. 1201, 321 (2009); 10.1063/1.3293066

The Cosmological Evolution of AGNs: Predictions from Galaxy Formation Models

AIP Conf. Proc. 1126, 215 (2009); 10.1063/1.3149416

Galaxy Evolution: What Do We Need To Know?

AIP Conf. Proc. 1035, 289 (2008); 10.1063/1.2973603 


\title{
Fragmentation in the First Galaxies
}

\author{
Chalence Safranek-Shrader \\ Department of Astronomy and Texas Cosmology Center, University of Texas at Austin, Austin, TX \\ 78712
}

\begin{abstract}
.
Using a one-zone model, we study the gravitational fragmentation of baryonic cold streams flowing into a typical first galaxy at high redshift to predict the expected fragmentation mass scale and thus a characteristic mass of the first population of stars to form by shock fragmentation. In this simplified model, we bypass the resolution barrier in simulations of progalaxy formation and directly probe the fragmentation mass scale of accreting gas streams. We find the fragmentation mass scale to be most strongly dependent on the metallicity of the gas and the background LymanWerner radiation field, and that either a large cluster of solar mass fragments or one large $\left(10^{5} M_{\odot}\right)$ fragment forms.
\end{abstract}

PACS: $98.80 .-\mathrm{k}$

\section{INTRODUCTION}

An important open question in cosmology is under which conditions the first Population II (Pop II) stars formed. It has been argued that clusters of predominantly low-mass stars could have arisen in the first galaxies at $z=10[1,2]$. Dense, metal-enriched baryonic streams flow along the filaments of the cosmic web and penetrate deep into a protogalaxy. The multiple streams collide with each other, or collide with a turbulentlysupported gas accumulation that has already collected in the center of the protogalaxy. Since the stream inflow is supersonic, the colliding gas must pass through a strong shock, but then cools rapidly in approximately isobaric conditions due to atomic cooling in the halo, whose virial temperature $T \sim 10^{4} \mathrm{~K}$.

\section{METHODOLOGY}

In order to properly model this shock fragmentation, we must accurately describe the chemical, thermal, and dynamical evolution of the gas flowing into a first galaxy, which we define to be a $10^{8} M_{\odot}$ dark matter (DM) halo at $z=10$. The cold stream shock heats to the virial temperature $\left(\sim 10^{4} \mathrm{~K}\right)$ near the center of the protogalaxy with a speed relative to the halo of order the virial velocity $\left(\sim 20 \mathrm{~km} \mathrm{~s}^{-1}\right)$. The post-shock region then cools and increases its density approximately isobarically [3]. From simulations [4], we assume that the pre-shock density of the gas is $10^{3} \mathrm{~cm}^{-3}$. 


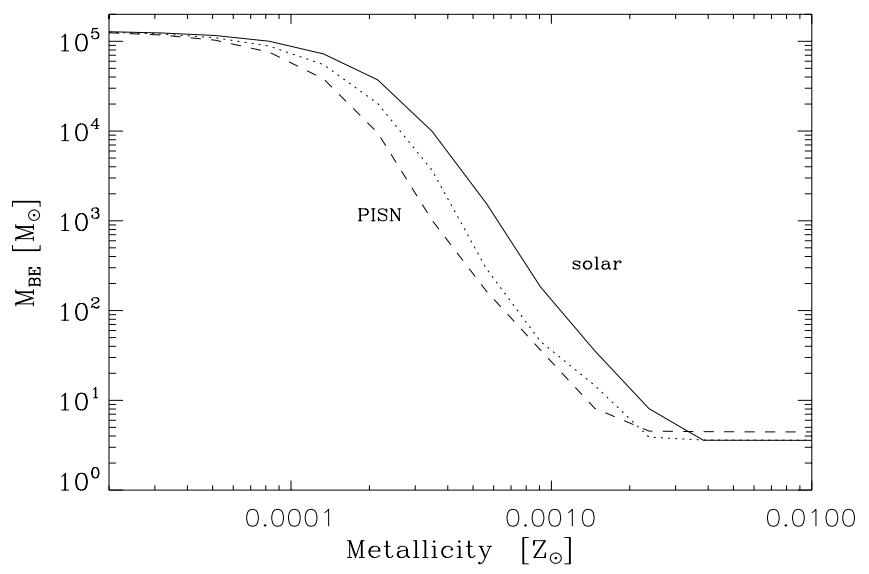

FIGURE 1. Fragmentation mass scale as a function of metallicity for $J_{21}=10^{4}$ (strong molecular suppression). At low metallicity, the gas is not able to cool past $T \sim 6000 \mathrm{~K}$ and thus has a mass scale set by initial conditions (density, virial temperature). For high metallicity, fine structure lines can cool the gas down to $T_{\mathrm{CMB}}$ which sets the BE mass, $\sim 1 \mathrm{M}_{\odot}$. The distinction between solar and PISN abundances is not significant.

\section{Chemical and Thermal Model}

We track the non-equilibrium chemical evolution of the gas starting from initial equilibrium abundances of species at $10^{4} \mathrm{~K}$. Helium abundances are set by BBN and we treat metallicity as a free parameter, with both solar and pair-instability supernova (PISN) abundances (neutral and singly ionized carbon, oxygen, silicon, and iron).

Cooling is from three main processes - ro-vibrational transitions in $\mathrm{H}_{2}$ (effective in the range $200 \mathrm{~K}<T<8000 \mathrm{~K}$ ), fine structure lines from neutral and singly ionized metals $(T<5000 \mathrm{~K})$, and atomic Lyman-alpha emission from neutral $\mathrm{H}\left(T>10^{4} \mathrm{~K}\right)$. Finally, the $\mathrm{CMB}$ temperature $T_{\mathrm{CMB}}=2.7 \mathrm{~K}(1+z)$ sets a lower limit to which gas can radiatively cool. We assume that the fragmentation mass is set by the local Bonnor-Ebert mass.

\section{RESULTS}

If no low temperature coolant is available (low metallicity and high $J_{21, \mathrm{LW}}$ ), the gas will not be able to cool and will fragment with a large BE mass, $10^{5} \mathrm{M}_{\odot}$. If, however, metals are present or $\mathrm{HD}$ is able to form, these will allow the gas to cool to $T_{\mathrm{CMB}}$ before the fragmentation criteria is reached, producing fragments with masses $\sim 1 \mathbf{M}_{\odot}$. Using a fit to the baryonic growth rate of halos [5], approximately $10^{5} \mathrm{M}_{\odot}$ of baryons enter the halo in the time it takes to fragment, suggesting either one large fragment or $\sim 10^{5}$ solar mass fragments as the end product of fragmentation, assuming $100 \%$ SF efficiency and that 
all baryonic mass enters through cold streams, neither of which in reality occur. Thus, these results suggest an upper limit to the size of the cluster and the mass of the resulting fragmentation byproducts. See Fig. 1 for the fragmentation mass scale as a function of metallicity.

\section{REFERENCES}

1. Bromm, V., \& Clarke, C. J. 2002, ApJL, 566, L1

2. Greif, T. H., \& Bromm, V. 2006, mnras, 373, 128

3. Shapiro, P.R., \& Kang, H. 1987, ApJ, 318, 32

4. Greif, T. H., Johnson, J. L., Klessen, R. S., \& Bromm, V. 2008, MNRAS, 387, 1021

5. Dekel, A., et al. 2009, Nature, 457, 451 